\title{
Quantifying the thickness of magnetically active layers using x-ray resonant magnetic scattering
}

\author{
B. M. Barnes, Z. Li, D. E. Savage, E. Wiedemann, and M. G. Lagallya) \\ University of Wisconsin-Madison, Madison, Wisconsin 53706 \\ (Presented on 6 January 2004)
}

\begin{abstract}
X-ray resonant magnetic scattering measurements below and above the critical angle for total external reflection allow fitting to extract the magnetically active volume within specific magnetic layers. Uncapped ultrathin $\mathrm{Co}$ films deposited onto $\mathrm{Ni}$ on $\mathrm{Si}$ display more magnetic resonant response than do those grown directly onto the native oxide of Si. (C) 2004 American Institute of Physics. [DOI: 10.1063/1.1667868]
\end{abstract}

Advances in magnetic storage and spin-based electronics require a thorough understanding of ferromagnetic-ferromagnetic and ferromagnetic-nonmagnetic interfaces. A ferromagnetic interface is a chemical boundary that may also serve as a magnetic boundary, delineating the magnetically active region of a layer. ${ }^{1}$ Recently, using diffuse soft-X-ray resonant magnetic scattering, (XRMS) or diffuse XRMS, we showed that the charge and magnetic morphologies are correlated for a $\mathrm{Fe} / \mathrm{Co}$ interface. ${ }^{2}$ At a vacuum/Co or an $\mathrm{Al} / \mathrm{Co}$ interface, the magnetic boundary differed in morphology from the charge interface. There is a region within the magnetic thin film that does not respond to an applied alternating magnetic field. The diffuse XRMS measurement quantified the morphology of the boundaries, but could not be used to specify the volume of the active magnetic layer, even where charge and magnetic morphologies are correlated. Information on the thickness of the magnetically active part of the magnetic thin film (and correspondingly the thicknesses of the nonactive parts) may be obtained through specular XRMS.

Following a report of a magnetically "dead" layer by Liebermann et al. ${ }^{3}$ in 1970 using Mössbauer spectroscopy, several techniques for measuring the thickness of nonmagnetic layers within nominally ferromagnetic films have been developed. These dead layers occur at the interfaces and may be thought of as transition layers between a foreign material interface and the magnetically active region. Therefore, surface-sensitive techniques are desired for measuring these layers. Some techniques, such as vibrating sample magnetometry, ${ }^{4}$ yield the active magnetic volume of the film but cannot separate surface from bulk properties. Scattering techniques are more sensitive to the interfaces of the film. Neutron scattering is an established and powerful tool for determining transition layers, 5,6 but, due to weak signals, this technique is best suited for studying multilayer structures with Bragg reflections. Soft x-ray techniques, such as X-ray

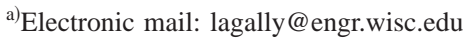

magnetic circular dichroism $(\mathrm{XMCD})^{7}$ and XRMS are best suited for measuring the transition layer for individual layers of transition metals. Similar in form to neutron reflectivity, ${ }^{8}$ XRMS has the added advantage of yielding structural information through the angular dependence of the scattered intensity. ${ }^{9}$ Also, XRMS is a photon in/photon out process, allowing measurements under applied magnetic fields ${ }^{10}$ unlike XMCD, which employs electrons for readout. The element-specific resonance at absorption edges also allows probing of one ferromagnetic element in the close proximity of a second ferromagnetic element.

Most reported measurements of XRMS are spectroscopic, measuring the resonant reflectivity as a function of incident photon energy. We follow the treatment of Raoux ${ }^{11}$ in measuring XRMS as a function of incident angle. Although total external reflection makes the relationship between probed depth and incident angle nonlinear, magnetic depth profiling may be possible using angularly dependent XRMS. To test this, three Co films ranging in thickness from 13 to $29 \AA$ (Table I) were deposited onto the native oxide of $\mathrm{Si}$ wafers, using dc magnetron sputtering at $2.0 \mathrm{mTorr} \mathrm{Ar}$ pressure in a UHV chamber with $\sim 1 \times 10^{-9}$ Torr. To study further the effect of an adjoining ferromagnetic film on the Co, three more Co films of approximately the same thickness were deposited onto $\sim 15 \AA$ of $\mathrm{Ni}$.

The samples are transferred without breaking the vacuum to a diffractometer chamber at the Synchrotron Radiation Center of the University of Wisconsin-Madison. ${ }^{12}$ For our XRMS measurements, we used a beamline with a $\mathrm{C} / \mathrm{W}$ multilayer mirror monochromator that delivers $\sim 6$ $\times 10^{8}$ elliptically polarized photons/s to the sample at $\sim 775 \mathrm{eV}$, the $L_{2}$ edge of Co. The energy resolution, $\Delta \lambda / \lambda$, is $4 \%$, or approximately $30 \mathrm{eV}$ at $775 \mathrm{eV}$. Experiments were performed at $E \sim 787 \mathrm{eV}$ as required by the aforementioned $4 \%$ energy resolution of the system. As the magnetically dependent dispersive optical constant, $\delta_{m}$, changes sign between the edges, it was valuable to reduce the influence of 
TABLE I. Surface roughness $\sigma_{\text {surface }}$, coercivity $H_{c}$, top and bottom dead layers $\Delta_{\text {top }}$ and $\Delta_{\text {bottom }}$, total nonmagnetic thickness $\Delta_{\text {top }}$, active layer thickness $d_{\text {active }}$ for Co sputter-deposited films, and asymmetry ratio above the critical angle for total external reflection. All thickness measurements determined by soft-x-ray fitting. Errors determined by confidence bars as in Fig. 2.

\begin{tabular}{|c|c|c|c|c|c|c|c|c|}
\hline Ferromagnetic layer $\pm d^{\text {err }}$ & Substrate & $\begin{array}{c}\sigma_{\text {surface }} \\
(\AA)\end{array}$ & $\begin{array}{c}H_{c} \\
(\mathrm{Oe})\end{array}$ & $\begin{array}{l}\Delta_{\text {top }} \\
(\AA)\end{array}$ & $\begin{array}{c}\Delta_{\text {bottom }} \\
(\AA)\end{array}$ & $\begin{array}{c}\Delta_{\text {total }} \\
(\AA)\end{array}$ & $d_{\text {active }}(\AA)$ & $\begin{array}{r}\mathrm{AR}(\%) \\
\theta @ 3^{\circ}\end{array}$ \\
\hline$(13 \AA \mathrm{Co} \pm 2)$ & $\mathrm{Si}$ & 4 & N/A & & 12.5 total & & $0.5 \pm 0.5$ & 1 \\
\hline$(13.5 \AA \mathrm{Co} \pm 4)$ & $14 \AA \mathrm{Ni} / \mathrm{Si}$ & 8 & 21 & 0.5 & 6.0 & 6.5 & $7.0 \pm 2.0$ & 9 \\
\hline$(16 \AA \mathrm{Co} \pm 2)$ & $\mathrm{Si}$ & 2 & 11 & 0.5 & 11.5 & 12.0 & $4.0 \pm 1.5$ & 7 \\
\hline$(27 \AA \mathrm{Co} \pm 4)$ & $14 \AA \mathrm{Ni} / \mathrm{Si}$ & 9 & 5 & 4.0 & 9.0 & 13.0 & $14.0 \pm 4.0$ & 12 \\
\hline$(29 \AA \mathrm{Co} \pm 3)$ & $\mathrm{Si}$ & 9 & 35 & 7.5 & 14.0 & 21.5 & $7.5 \pm 2.5$ & 7 \\
\hline$(40 \AA \mathrm{Co} \pm 7)$ & $14 \AA \mathrm{Ni} / \mathrm{Si}$ & 12 & 11 & 10.0 & 7.5 & 17.5 & $22.5 \pm 5.0$ & 13 \\
\hline
\end{tabular}

the $L_{3}$ edge at $778 \mathrm{eV}$ and increase the contribution of the $L_{2}$ edge at $793 \mathrm{eV}$.

We measure the scattered $\mathrm{x}$ rays as a function of the incident angle $\theta$ and detector angle $2 \theta$ (Fig. 1) to obtain the specularly reflected intensity. We augment this $\theta-2 \theta$ scan with fixed-detector rocking curves at each detector position $2 \theta$ to help our custom-built diffractometer maintain synchronization with the reflection and provides background scattering information at the scan extrema. In addition to the XRMS measurements, we obtain element specific hysteresis curves using the XRMS specular reflection to determine the coercive force and magnetic quality of the films. These scans are done at a fixed $\theta=3^{\circ}$ angle of incidence, maximizing reflectance by staying just off the regime of total external reflection.

We magnetize the sample with an electromagnet that can deliver a maximum field of $\sim 64 \mathrm{G}$ without outgassing into the vacuum chamber, either parallel or antiparallel to the photon helicity. We generally use fields of $\sim 55 \mathrm{G}$, which is greater than the coercive field of our samples. We measure the scattered intensity point by point for magnetization parallel and antiparallel to the photon helicity, called $I_{+}$and $I_{-}$, respectively. We then calculate the difference intensity, $\Delta I(\mathbf{M}, \Omega)=I_{+}-I_{-}$, and the sum intensity, $I_{\text {ave }}(\theta)=(1 / 2)$ $\times\left(I_{+}+I\right)$. A scan from a demagnetized sample would have an intensity of $(1 / 2)\left(I_{+}+I_{-}\right)$, i.e., between a full scan taken with magnetization parallel to the photon helicity, and a scan taken with the magnetization antiparallel. $\Delta I(\mathbf{M}, \theta)$ and $I_{\text {ave }}(\theta)$ are then divided by the initial incident intensity at $\theta$ $=0$, yielding the a nonresonant reflectivity, $R$, and a resonant difference reflectivity, $\Delta R$.

We use the reflectivity and difference reflectivity to measure the asymmetry ratio, the intensity when the sample is magnetized in one direction minus the intensity when the sample is magnetized in the opposite direction, divided by their average. Its value for a given element depends on the relative number of moments that follow the applied field. In

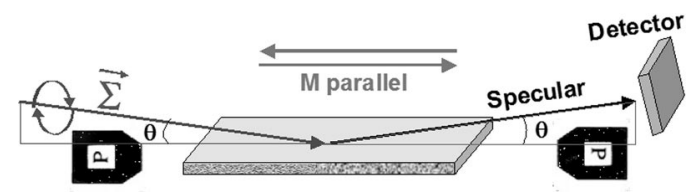

FIG. 1. Scattering geometry for specular XRMS measurements. At every detector angle $2 \theta$, we measure a rocking curve. We correct for the background using points away from the specular reflection. the limit that a film is nonmagnetic or for some reason nonresponsive, the asymmetry ratio is zero, i.e., there is no change in intensity when the direction of the field is reversed. Values for the asymmetry ratio at $\theta=3^{\circ}$ are also given in Table I.

Element-specific hysteresis curves (not shown) of the bare-Co/Si film yield a nearly square hysteresis loop, indicating a single-domain sample, and a coercive field of about $11 \mathrm{G}$. The $\mathrm{Co} / \mathrm{Ni} / \mathrm{Si}$ film shows lower squareness and may have multiple domains. It has a coercive field of about $5 \mathrm{G}$. The low coercivity values indicate that the films have a low defect density, since lower coercivity generally indicates fewer pinning centers for magnetic domains. ${ }^{13}$ Coercivities for all samples may be found in Table I.

The reflectivity and difference reflectivity were fitted to obtain the active and transition layer thicknesses. Calculations were performed using the recursive matrix algorithm code for resonant reflectivity of Lee et al. ${ }^{14}$ We interpolated nonresonant optical constants from the tables of Henke et $a l .{ }^{15}$ and resonant optical constants from Mertins et al., ${ }^{16}$ multiplying the latter by 0.70 to approximate the incomplete circular polarization expected from synchrotron radiation. Although this code allows a full treatment of resonant and nonresonant contributions and their interactions with the circularly polarized beam, we chose to simplify modeling by

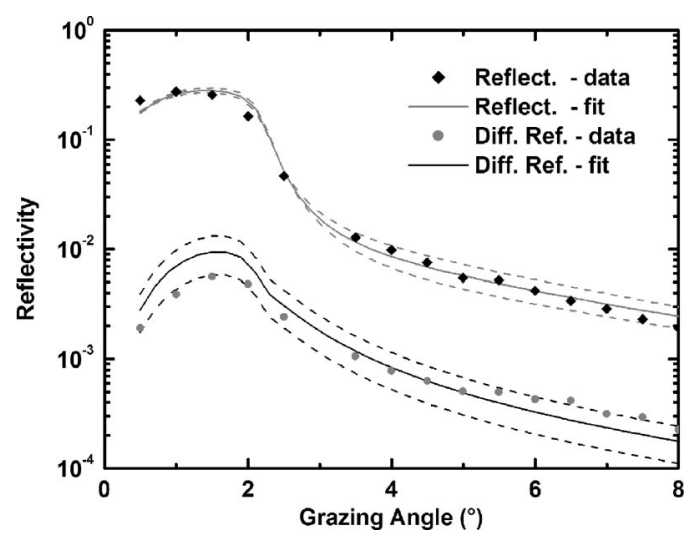

FIG. 2. Example of reflectivity and difference reflectivity fits for the $16 \AA$ Co film on Si. Dashed lines represent the $\pm 2 \AA$ uncertainty in the reflectivity fit and the corresponding $\pm 1.5 \AA$ uncertainty in the difference reflectivity fit. The finite beam width and finite sample height are included in the fit to correct discrepancies at small angles above the critical angle. Some curves have higher uncertainty or only fit above the critical angle for total external reflection. See Table I for values. 


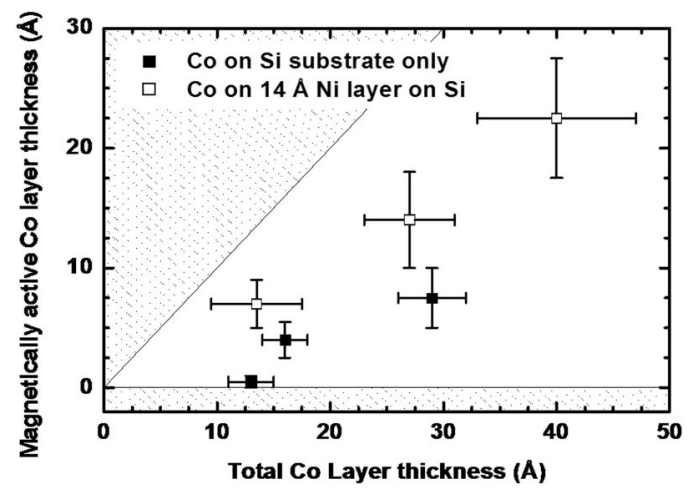

FIG. 3. Comparison between the active magnetic-layer thickness and the total magnetic-layer thickness for $\mathrm{Co}$ on $\mathrm{Si}$ and $\mathrm{Co}$ on $\mathrm{Ni} / \mathrm{Si}$. The shaded regions are not physical. The difference reflectivity from the $13 \AA$ Co film on $\mathrm{Si}$ was negligible. The Co films on Ni exhibited more element-specific difference reflectivity than did the Co films on $\mathrm{Si}$, possibly due to the reinforcement of the easy in-plane axis by the $\mathrm{Ni}$.

folding all magneto-optical effects into an extra term in the index of refraction, ${ }^{6}$

$$
n_{ \pm}=1-(\delta \pm \Delta \delta)+i(\beta \pm \Delta \beta),
$$

where $\delta, \beta$ are the nonresonant dispersive and absorptive components of the index of refraction, and $\Delta \delta, \Delta \beta$ are the resonant dispersive and absorptive terms, which change sign with the direction of the applied field. Our rationale is that our stated energy resolution, $\Delta \lambda / \lambda, 4 \%$, necessitates numerically integrating fits over a range of energies near the $L_{2}$ edge, complicating our fitting. An example fit to the data, with dotted lines to represent confidence intervals, is shown in Fig. 2. The fits are optimized for the angular region at and just past the critical angle for total external reflection. Fit results are summarized also in Table I.

Figure 3 shows the full results of the fitting of the magnetically active Co film thickness as a function of total Co film thickness for both types of substrates. We can clearly resolve that the addition of $\mathrm{Ni}$ as an underlayer to thin $\mathrm{Co}$ films makes the apparent Co magnetic volume increase and the Co transition layer thickness decrease. A previous study of evaporated $\mathrm{Co}$ on $\mathrm{Si}(111)^{17}$ showed that 3 monolayer (ML) thick films of Co were nonmagnetic, and that films 3 ML to 9 ML in thickness were magnetic with the easy axis canted away from the sample plane. The apparent increase in ferromagnetic volume relative to $\mathrm{Co}-\mathrm{on}-\mathrm{Si}$ due to the $\mathrm{Ni}$ underlayer may be due to the Ni changing the angle of the canting more toward an easy in-plane axis. As XRMS measures only in-plane spins, any tilting toward the sample plane would increase the resonant scattering. Even if the Ni does not change the orientation of the magnetically active spins, the Ni may provide coordination to nominally uncoordinated Co spins at the interface. Other explanations are also possible, e.g., differing amounts of intermixing between $\mathrm{Co} / \mathrm{Ni}$ and $\mathrm{Co} / \mathrm{Si}$. This element-specific measurement includes the effect of the $\mathrm{Ni}$ on the $\mathrm{Co}$, without including any resonant contribution from the Ni itself.

Soft-x-ray angular-dependent XRMS is an effective tool for measuring the in-plane magnetization of thin ferromagnetic films. The in-plane magnetization of $10-30 \AA$ thick Co on $\mathrm{Si}$ was less than that for similar depositions of $\mathrm{Co}$ on $\sim 15 \AA$ of $\mathrm{Ni}$. The fitting indicates that the $\mathrm{Co} / \mathrm{Si}$ interface creates a larger magnetic transition layer than $\mathrm{Co} / \mathrm{Ni}$ or vacuum/Co interfaces. The element-specific nature of XRMS allows quantification of the active volume within the Co layer and holds promise as an in-plane magnetic-depth profile tool, and as a complementary tool to absorption techniques, such as XMCD, that may be performed out of plane.

This work was supported by ONR. The authors thank D. R. Lee for use of his code ${ }^{14}$ and thank D. R. Lee and S. Stepanov for their helpful suggestions. This work was performed at the Synchrotron Radiation Center, UW-Madison, funded by the NSF under Award No. DMR-0084402.

${ }^{1}$ R. M. Osgood, III, S. K. Sinha, J. W. Freeland, Y. U. Idzerda, and S. D. Bader, J. Magn. Magn. Mater. 198, 698 (1999).

${ }^{2}$ J. J. Kelly, IV, B. M. Barnes, F. Flack, D. P. Lagally, D. E. Savage, M. Friesen, and M. G. Lagally, J. Appl. Phys. 91, 9978 (2002).

${ }^{3}$ L. Liebermann, J. Clinton, D. M. Edwards, and J. Mathon, Phys. Rev. Lett. 25, 232 (1970).

${ }^{4}$ S. N. Shringi, S. N. Piramanayagam, S. Prassad, N. Venkatramani, R. Krishnan, and M. Tessier, J. Appl. Phys. 73, 6438 (1993).

${ }^{5}$ G. P. Felcher, Phys. Rev. B 24, 1595 (1981).

${ }^{6}$ J. F. Ankner, C. F. Majkrzak, and H. Homma, J. Appl. Phys. 73, 6436 (1993).

${ }^{7}$ W. L. O’Brien and B. P. Tonner, Phys. Rev. B 52, 15332 (1995).

${ }^{8}$ S. S. P. Parkin, R. Sigsbee, R. Felici, and G. P. Felcher, Appl. Phys. Lett. 48, 604 (1986).

${ }^{9}$ C.-C. Kao, C. T. Chen, E. D. Johnson, J. B. Hastings, H. J. Lin, G. H. Ho, G. Meigs, J.-M. Brot, S. L. Hubert, Y. U. Idzerda, and C. Vettier, Phys. Rev. B 50, 9599 (1994).

${ }^{10}$ M. Sacchi and A. Mirone, Phys. Rev. B 57, 8408 (1998).

${ }^{11}$ D. Raoux, N. Jaouen, J. M. Tonnerre, and E. Bontempi, Acta Phys. Pol. A 98, 483 (2000).

${ }^{12}$ J. F. MacKay, D. W. Pearson, B. E. Nelms, P. M. DeLuca, Jr., M. N. Gould, and M. G. Lagally, Med. Phys. 25, 773 (1998)

${ }^{13}$ K. N. Altmann, J. A. Con Foo, F. J. Himpsel, J. J. Kelly, M. G. Lagally, J. F. MacKay, W. L. O'Brien, J. E. Ortega, and D. Y. Petrovykh, Mater. Res. Soc. Symp. Proc. 570, 141 (1999), and references therein.

${ }^{14}$ D. R. Lee, S. K. Sinha, D. Haskel, Y. Choi, J. C. Lang, S. A. Stepanov, and G. Srajer, Phys. Rev. B 68, 224409 (2003).

${ }^{15}$ B. L. Henke, E. M. Gullikson, and J. C. Davis, At. Data Nucl. Data Tables 54, 181 (1993).

${ }^{16}$ H.-C. Mertins, F. Schaefers, X. Le Cann, A. Gaupp, and W. Gudat, Phys. Rev. B 61, R874 (2000).

${ }^{17}$ J.-S. Tsay and Y.-D. Yao, Appl. Phys. Lett. 74, 1311 (1999). 